\title{
Correction to: Combined viral quasispecies diversity and hepatitis $B$ core-related antigen predict off-nucleos(t)ide analog durability in $\mathrm{HBeAg}$-negative patients
}

\author{
Huei-Ru Cheng ${ }^{1} \cdot$ Hung-Chih Yang ${ }^{1,2,3} \cdot$ Su-Ru Lin ${ }^{1} \cdot$ Ta-Yu Yang $^{2} \cdot$ You-Yu Lin ${ }^{1} \cdot$ Tung-Hung Su ${ }^{3,4} \cdot$ Tai-Chung Tseng ${ }^{3,4}$. \\ Chun-Jen Liu ${ }^{3,4}$. Jia-Horng Kao ${ }^{1,3,4}$
}

Published online: 25 September 2021

(c) Asian Pacific Association for the Study of the Liver 2021

\section{Correction to: Hepatology International (2021) 15:582-592 https://doi.org/10.1007/s12072-021-10186-7}

Unfortunately, Fig. 3c and d were erroneously labeled in the original publication of the article.

In Fig. 3c, the number of nucleotide diversity at the bottom left corner should be 0.01 instead of 1.00. In Fig. 3d, the group with nucleotide diversity/HBcrAg " $<0.01$ and $\geqq$

2.00 " should be corrected as " $<0.01$ or $\geqq 2.00$ ". Although these two figures were erroneously labeled, all the relevant results in the text were correctly stated. The correct Fig. 3 are given below:

The original article can be found online at https://doi.org/10.1007/ s12072-021-10186-7.

Jia-Horng Kao

kaojh@ntu.edu.tw

1 Department of Internal Medicine, Graduate Institute of Clinical Medicine, National Taiwan University College of Medicine, 1 Chang-Te St., Taipei 10002, Taiwan

2 Department of Microbiology, National Taiwan University College of Medicine, Taipei, Taiwan

3 Division of Gastroenterology and Hepatology, Department of Internal Medicine, National Taiwan University Hospital, Taipei, Taiwan

4 Hepatitis Research Center, National Taiwan University Hospital, Taipei, Taiwan 
A

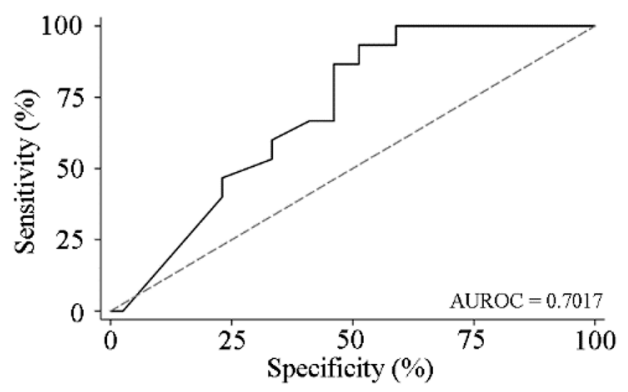

C

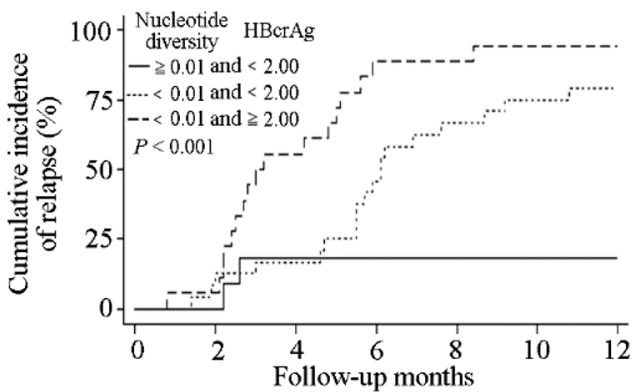

Nucleotide HBerAg Number at risk

$\begin{array}{lccccccc}\geqq 0.01 \text { and }<2.00 & 11 & 11 & 9 & 9 & 9 & 9 & 8 \\ <0.01 \text { and }<2.00 & 24 & 22 & 20 & 13 & 8 & 6 & 4 \\ <0.01 \text { and } \geqq 2.00 & 18 & 17 & 8 & 2 & 2 & 1 & 1\end{array}$

Fig. 3 Pretreatment viral genetic diversity of the precore/core gene and HBcrAg predicts the risk of virological relapse after discontinuation of NAs. a The AUROC analysis of HBcrAg in prediction of virological relapse within 1-year off-therapy. $\mathbf{b}$ The cumulative incidence of 1 -year virological relapse in patients with low $(<2 \mathrm{KU} / \mathrm{mL})$ and high ( $\geqq 2 \mathrm{KU} / \mathrm{mL}$ ) levels of baseline HBcrAg. $\mathbf{c}$ The cumulative inci-
B

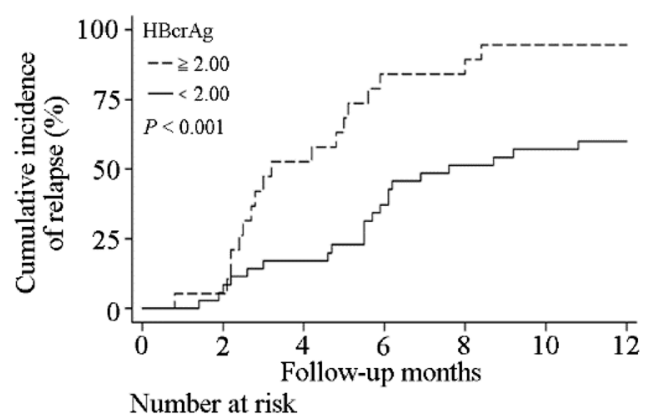

HBcrAg $\begin{array}{rrrrcccc}\geqq 2.00 & 19 & 18 & 9 & 3 & 3 & 1 & 1 \\ <2.00 & 35 & 33 & 29 & 22 & 17 & 15 & 12\end{array}$

D

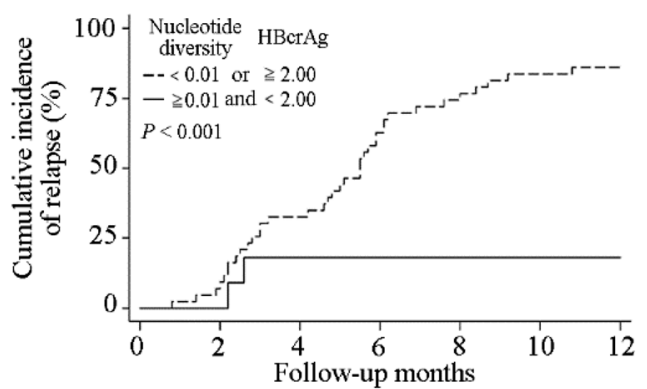

Nucleotide
diversity

$\begin{array}{lllccccc}<0.01 \text { or } \geqq 2.00 & 43 & 40 & 29 & 16 & 11 & 7 & 5 \\ \geqq 0.01 \text { and }<2.00 & 11 & 11 & 9 & 9 & 9 & 9 & 8\end{array}$

dence of 1-year virological relapse in three groups of patients with different levels of baseline the viral nucleotide diversity and HBcrAg. d The cumulative incidence of 1-year virological relapse in two groups of patients with different levels of baseline the viral nucleotide diversity and $\mathrm{HBcrAg}$

Publisher's Note Springer Nature remains neutral with regard to jurisdictional claims in published maps and institutional affiliations. 